\title{
A novel role for the Pol I transcription factor UBTF in maintaining genome stability through the regulation of highly transcribed Pol II genes
}

\author{
Elaine Sanij, ${ }^{1,2,3}$ Jeannine Diesch, ${ }^{1,2}$ Analia Lesmana, ${ }^{1,2}$ Gretchen Poortinga, ${ }^{1,2,4}$ \\ Nadine Hein, ${ }^{1,2}$ Grace Lidgerwood, ${ }^{1}$ Donald P. Cameron, ${ }^{1,2}$ Jason Ellul, ${ }^{1,2}$ \\ Gregory J. Goodall, ${ }^{5,6,7}$ Lee H. Wong, ${ }^{8}$ Amardeep S. Dhillon, ${ }^{1,2,3}$
} Nourdine Hamdane, ${ }^{9,10}$ Lawrence I. Rothblum, ${ }^{11}$ Richard B. Pearson, ${ }^{1,2,8,12}$ Izhak Haviv, ${ }^{3,13,16}$ Tom Moss, ${ }^{9,10}$ and Ross D. Hannan $1,2,8,12,14,15,16$

${ }^{1}$ Research Division, Peter MacCallum Cancer Centre, East Melbourne, Victoria 3002, Australia; ${ }^{2}$ Sir Peter MacCallum Department of Oncology, University of Melbourne, Parkville, Victoria 3010, Australia; ${ }^{3}$ Department of Pathology, University of Melbourne, Parkville, Victoria 3010, Australia; ${ }^{4}$ Department of Medicine, University of Melbourne, Parkville, Victoria 3010, Australia; ${ }^{5}$ Centre for Cancer Biology, SA Pathology and University of South Australia, Adelaide, South Australia 5000, Australia; ${ }^{6}$ Discipline of Medicine, The University of Adelaide, Adelaide, South Australia 5005, Australia; ${ }^{7}$ School of Molecular and Biomedical Science, The University of Adelaide, Adelaide, South Australia 5005, Australia; ${ }^{8}$ Department of Biochemistry and Molecular Biology, Monash University, Clayton, Victoria 3800, Australia; ${ }^{9}$ Department of Molecular Biology, Medical Biochemistry and Pathology, Faculty of Medicine, Laval University, Québec, QC, G1V 0A6, Canada; ${ }^{10}$ St-Patrick Research Group in Basic Oncology, Québec University Hospital Research Centre, Québec, QC, G1R 3S3, Canada; ${ }^{11}$ Department of Cell Biology, University of Oklahoma College of Medicine, Oklahoma City, Oklahoma 73104, USA; ${ }^{12}$ Department of Biochemistry and Molecular Biology, University of Melbourne, Parkville, Victoria 3010, Australia; ${ }^{13}$ Faculty of Medicine, Bar-Ilan University, Zfat, 13100, Israel; ${ }^{14}$ Division of Cancer Medicine, Peter MacCallum Cancer Centre, East Melbourne, Victoria 3002, Australia; ${ }^{15}$ School of Biomedical Sciences, University of Queensland, Brisbane 4072, Queensland, Australia

\begin{abstract}
Mechanisms to coordinate programs of highly transcribed genes required for cellular homeostasis and growth are unclear. Upstream binding transcription factor (UBTF, also called UBF) is thought to function exclusively in RNA polymerase I (Pol I)-specific transcription of the ribosomal genes. Here, we report that the two isoforms of UBTF (UBTF1/2) are also enriched at highly expressed Pol II-transcribed genes throughout the mouse genome. Further analysis of UBTF1/2 DNA binding in immortalized human epithelial cells and their isogenically matched transformed counterparts reveals an additional repertoire of UBTF1/2-bound genes involved in the regulation of cell cycle checkpoints and DNA damage response. As proof of a functional role for UBTF1/2 in regulating Pol II transcription, we demonstrate that UBTF1/2 is required for recruiting Pol II to the highly transcribed histone gene clusters and for their optimal expression. Intriguingly, lack of UBTF1/ 2 does not affect chromatin marks or nucleosome density at histone genes. Instead, it results in increased accessibility of the histone promoters and transcribed regions to micrococcal nuclease, implicating UBTF1/2 in mediating DNA accessibility. Unexpectedly, UBTF2, which does not function in Pol I transcription, is sufficient to regulate histone gene expression in the absence of UBTF1. Moreover, depletion of UBTF1/2 and subsequent reduction in histone gene expression is associated with DNA damage and genomic instability independent of Pol I transcription. Thus, we have uncovered a novel role for UBTF1 and UBTF2 in maintaining genome stability through coordinating the expression of highly transcribed Pol I (UBTF1 activity) and Pol II genes (UBTF2 activity).
\end{abstract}

[Supplemental material is available for this article.]

Transcription of ribosomal RNA (rRNA) genes (rDNA) is mediated by RNA polymerase I (Pol I) and its associated transcription machinery to produce the $45 \mathrm{~S}$ rRNA precursor of the $28 \mathrm{~S}, 5.8 \mathrm{~S}$, and $18 \mathrm{~S}$ rRNA components of the ribosome (McStay and Grummt 2008). In rapidly growing mammalian cells, rRNA synthesis accounts for $35 \%-60 \%$ of all RNA transcription (Moss and Stefanovsky 2002;

\footnotetext{
${ }^{16}$ These authors contributed equally to this work. Corresponding authors: elaine.sanij@petermac.org, ross.hannan@ petermac.org

Article published online before print. Article, supplemental material, and publication date are at http://www.genome.org/cgi/doi/10.1101/gr.176115.114. Freely available online through the Genome Research Open Access option.
}

Cavanaugh et al. 2003). Accordingly, the Pol I transcription rate is tightly coupled to cellular growth and proliferation rates (Hannan and Rothblum 1995; Moss and Stefanovsky 2002; McStay and Grummt 2008; Chan et al. 2011; Hein et al. 2012; Diesch et al. 2014). We have shown that the consistent up-regulation of rRNA synthesis in cancer cells is necessary for malignant transformation in certain settings (Drygin et al. 2011; Bywater et al. 2012), while small molecule inhibitors of Pol I transcription are currently in

(C) 2015 Sanij et al. This article, published in Genome Research, is available under a Creative Commons License (Attribution-NonCommercial 4.0 International), as described at http://creativecommons.org/licenses/by-nc/4.0/. 
preclinical development and phase I trials for cancer therapy (Hein et al. 2013; Drygin et al. 2014; Poortinga et al. 2014). Despite this, the exact molecular mechanism by which Pol I transcription is coordinated with Pol II transcriptional programs to ensure orderly cellular homeostasis is not fully understood. In this report, we propose a functional role for the Pol I-specific transcription factor UBTF1/2 in coordinating Pol I-mediated rDNA transcription with expression of highly active Pol II-transcribed genes.

UBTF1/2 belongs to the sequence nonspecific class of HMG (high mobility group) proteins. It has six HMG-box homology domains and has been proposed to interact with DNA as a dimer and to induce six in-phase bends to generate a single $360^{\circ}$-loop structure that resembles the nucleosome (Stefanovsky et al. 2001). UBTF1/2 binds across the transcribed and control regions of the rRNA genes (Supplemental Fig 1A; O'Sullivan et al. 2002; Sanij and Hannan 2009), probably explaining its multiple roles in preinitiation complex (PIC) formation, transcription initiation, and elongation (for review, see Moss et al. 2007; McStay and Grummt 2008; Sanij and Hannan 2009). Indeed, total ablation of $U b t f 1 / 2$ eliminates all rRNA gene transcription and leads to changes in rDNA chromatin (Hamdane et al. 2014).

We have previously shown that UBTF1, but not the naturally occurring splice variant UBTF2, is essential in determining and maintaining the euchromatic state of active rDNA in mammalian cells (Sanij et al. 2008). Further, we have reported that depletion of Ubtf1/2 by RNA interference (RNAi) silences active rRNA genes and is associated with histone H1-induced assembly of transcriptionally inactive rDNA chromatin (Sanij et al. 2008). Indeed, UBTF1/2 localizes with decondensed active rDNA, while inactive rDNAs appear as bright dense loci devoid of UBTF1/2 (Supplemental Fig. 1B) that are enhanced in number by Ubtf1/2 loss (Hamdane et al. 2014). This is in agreement with its reported function in establishing and maintaining the undercondensed structure of active nucleolar organizer regions (NORs), which is required for rDNA transcription and nucleolar assembly (Mais et al. 2005; Sanij et al. 2008; Sanij and Hannan 2009; Grob et al. 2014; Hamdane et al. 2014).

Intriguingly, UBTF2 was identified in a functional screen as an enhancer of the beta-catenin pathway (Grueneberg et al. 2003). Further, UBTF1/2 was noted to be putatively enriched at Pol II genes across the human genome (Zentner et al. 2011). However, the role of extra-nucleolar UBTF1/2 is unknown. Indeed, the prevailing consensus in the transcription field is still overwhelmingly that UBTF1/2 is exclusively a Pol I transcription factor (Grob et al. 2014). Here we demonstrate that in addition to the Pol I loci, UBTF1/2 is enriched at and regulates highly expressed Pol II-transcribed genes including histone gene clusters. Further, ChIP-seq analysis of UBTF1/2 binding in tumorigenic human epithelial cells compared to isogenically matched primary cells reveals an additional repertoire of UBTF1/2 target genes involved in regulation of cell cycle checkpoints and DNA damage response. Depletion of $U b t f 1 / 2$ by RNAi leads to DNA damage and genomic instability independent of Pol I transcription. Together, our data demonstrate a novel and unexpected role for UBTF1 and UBTF2 in coordinating Pol I-mediated rDNA transcription with expression of distinct sets of highly active Pol II-transcribed genes that control cell cycle progression and genome integrity.

\section{Results}

\section{UBTF1/ 2 binds highly active Pol Il-transcribed genes}

To identify a species and cell type-independent core set of UBTF1/2 target genes, we performed ChIP-seq analysis in mouse NIH3T3 cells (post-crisis, immortalized embryonic fibroblasts) and the human primary mammary epithelial cell line (HMEC) immortalized by expressing TERT, the catalytic subunit of telomerase (Elenbaas et al. 2001). We identified, respectively, 3705 and 10,726 genomic regions with significant UBTF1/2 enrichment across the mouse and human genomes, of which, respectively, $46 \%$ and $52 \%$ overlapped any exon/intron of known RefSeq genes (Fig. 1A,B). In both mouse and human cells, $\sim 40 \%$ of all UBTF1/2 binding overlapped with first exons and introns of annotated genes. Indeed, UBTF1/2 enrichment shows preference for binding near transcription start sites (TSSs), whereas no significant binding preference was observed at the transcription termination sites (TTSs) (Fig. 1C). Gene ontology analysis using the Genomic Regions Enrichment of Annotation Tool (GREAT) (McLean et al. 2010) identified chromatin assembly and nucleosome organization and assembly as the biological processes most significantly enriched with UBTF1/2-bound genes and common between the mouse and human data sets (Supplemental Fig. 2A,B). These categories include chromatin remodeling factors and, significantly, a large number of histone genes (Supplemental Tables 1, 2), implicating a role for UBTF1/2 in their transcriptional regulation. To confirm UBTF1/2 enrichments at these non-rDNA loci identified by ChIP-seq, we examined UBTF1/2 DNA binding manually by quantitative ChIP (qChIP) at genes encoding histone genes, chromatin remodeling factors (Asf1a, Smarca5, and Dnmt3a), and also potential UBTF1/2 target genes that did not appear within gene ontological categories, e.g., deoxyuridine triphosphatase (Dut), the oncogene $M y c$, and the member of the structural maintenance of chromosome family (Smc4) (Fig. 1D). The qChIP data showed significant enrichment in UBTF1/2 at these genes as compared to controls, including the intergenic spacer (IGS) of rDNA that does not bind UBTF1/2 (Fig. 1D).

We intersected the lists of annotated genes bound by UBTF1/2 within $\pm 2 \mathrm{~kb}$ of TSSs in the NIH3T3 and HMEC data sets and identified 699 genes that were common between the mouse and human analyses (Fig. 1E; Supplemental Table 14). Gene ontology analysis of the intersected genes showed regulation of gene silencing, chromatin, and nucleosome assembly categories to be the most significantly enriched with UBTF1/2-bound genes (Supplemental Fig. 2C). The gene lists for these ontology terms consisted mainly of histone genes and chromatin remodeling factors (Supplemental Tables 3-5), suggesting a conserved function for UBTF1/ 2 in their transcriptional regulation. Given that histone genes are among the most highly expressed genes in the human genome (Anamika et al. 2012), we investigated whether UBTF1/2 binding correlates with transcriptional activity. Indeed, a correlation of UBTF1/2 DNA binding with global gene expression in NIH3T3 cells identified genes enriched with UBTF1/2 at their TSSs to be expressed at high levels compared to non-UBTF1/2-bound genes and all genes in the genome (Fig. 1F).

We next examined the enrichments of a variety of posttranslational histone modifications, Pol II binding, and DNase I hypersensitivity (HS) profiles at UBTF1/2-bound regions in NIH3T3 cells (Fig. 2A). We observed little correlation between UBTF1/2 binding and the presence of the transcriptional repressive mark H3K9me3. However, almost 50\% of the UBTF1/2 peaks overlapped with the activating $\mathrm{H} 3 \mathrm{~K} 4 \mathrm{me} 3, \mathrm{H} 4 \mathrm{~K} 9 \mathrm{ac}$, and $\mathrm{H} 4$ hyperacetylation marks and Pol II enrichment. Further, almost 70\% of UBTF1/2bound regions are defined as DNase I hypersensitive sites, indicative of open and accessible chromatin (Fig. 2A). Thus, our data strongly suggest specific preference for UBTF1/2 binding to open chromatin structures associated with active promoters and gene bodies.

\section{Genome Research}

www.genome.org 
A

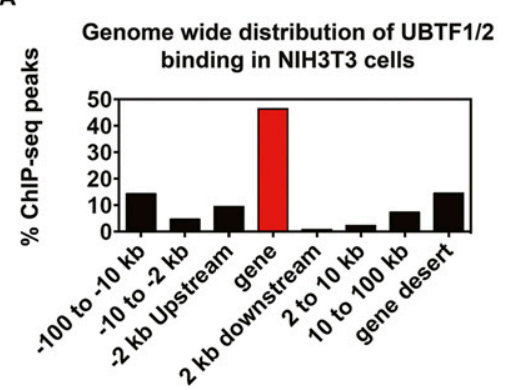

C

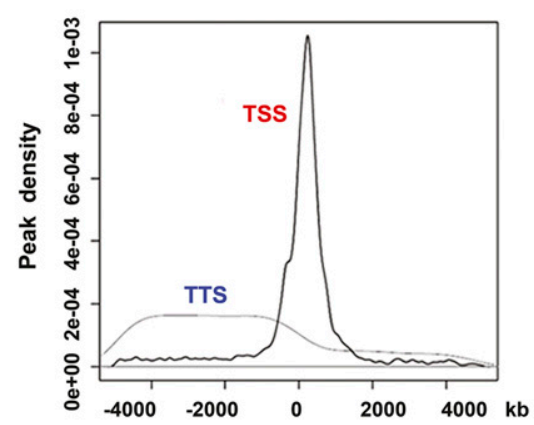

E

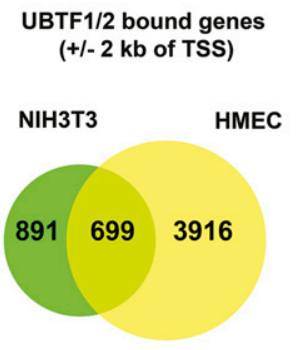

B

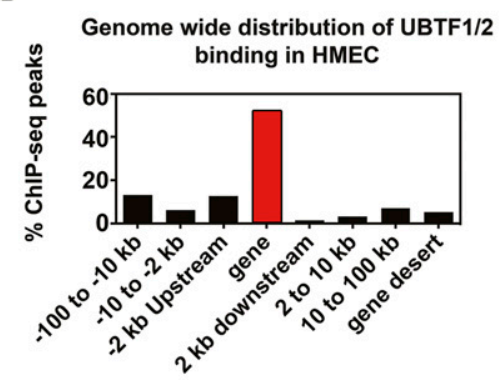

D

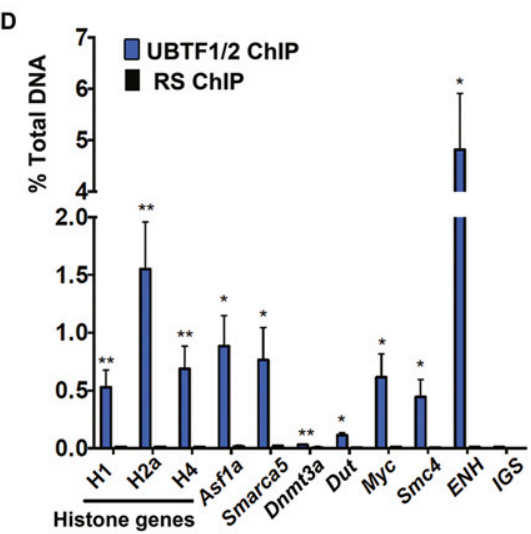

$\mathbf{F}$

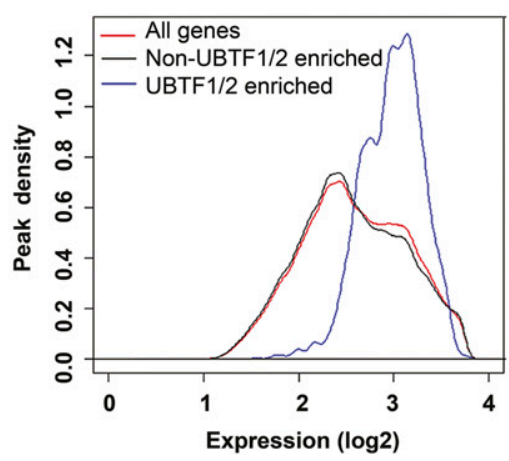

peaks of Pol I binding across the mouse and human genomes, some of which we validated manually by qChIP (Supplemental Fig. 3A). However, a comparison of UBTF1/2 and POLR1A ChIP-seq analysis in NIH3T3 and HMEC cell lines revealed little overlap in binding (less than $8 \%$ and 3\%) across the mouse and human genomes, respectively (Fig. 2B). Thus the data demonstrate that the Pol I transcription apparatus does not simply colocalize indiscriminately at open chromatin and that the enrichment at classes of genes involved in chromatin assembly and nucleosome organization is specific to UBTF1/2. Further, the data strongly suggest that UBTF $1 / 2$ does not recruit Pol I to Pol II genes.

Importantly, gene ontology analysis on the intersected UBTF1/2- and Pol II-bound regions revealed negative regulation of megakaryocyte differentiation, chromatin assembly, and regulation of gene silencing to be the biological processes most significantly enriched (Supplemental Fig. 2D). The UBTF1/2-bound gene lists belonging to these ontology terms consisted mainly of chromatin remodeling factors and histone genes (Supplemental Tables 6-9). Thus, our data, collectively along with the known role of UBTF1/2 in regulating rRNA gene transcription, suggest that UBTF1/2 is required for transcription of distinct classes of highly expressed genes involved in cellular proliferative growth, including regulators of chromatin organization.

UBTF1/ 2 are enriched at a novel subset of Pol II genes in transformed human cells

To determine whether malignant transformation is associated with altered UBTF1/2 enrichment at Pol II genes, we compared UBTF1/2 enrichment in the HMECs (Fig. 1B) to UBTF1/2 enrichment in the tumorigenic HMLER cells, an isogenic HMECderived cell line expressing the SV40 large-T, TERT, and an oncogenic allele of the HRAS gene (expressing HRAS $^{\mathrm{V} 12 \mathrm{G}}$ ) that forms tumors in nude mice (Elenbaas et al. 2001). We identified 11,990 genomic regions with UBTF $1 / 2$ enrichment. We next intersected the UBTF1/2 ChIP-

Interestingly, UBTF1/2 ChIP-seq peaks account for only $1.7 \%$ of all DNase I HS regions across the genome (Fig. 2A). This suggests that UBTF1/2 preferentially binds a specific subclass of open highly transcribed chromatin. In order to confirm the specificity of UBTF1/2 binding to DNA, we performed ChIP-seq analysis of POLR1A, the largest subunit of the RNA Pol I complex as both Pol I and UBTF1/2 co-occupy the highly transcribed Pol I-dependent rDNA loci (Supplemental Fig. 1A). We detected 1409 and 9296 seq data in HMECs and HMLERs to identify common and unique regions of UBTF1/2 enrichment in HMLERs (Fig. 2C; Supplemental Fig 2E,F) and identified 3687 UBTF1/2-bound genomic sequences common to both the primary and the tumorigenic cell lines, with genes encoding chromatin and nucleosome assembly being among the most overrepresented (Supplemental Fig. 2E; Supplemental Table 10). Thus the regulation of histone and chromatin remodeling genes is a fundamentally conserved function of 
A
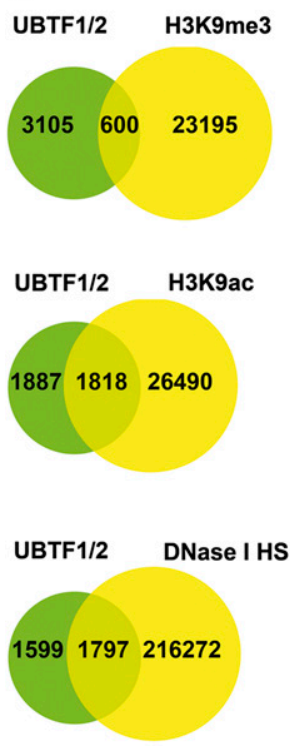

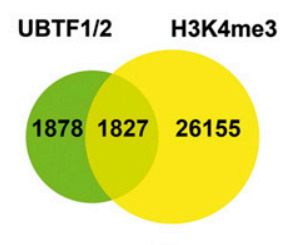

H4

UBTF1/2 hyperacetylation
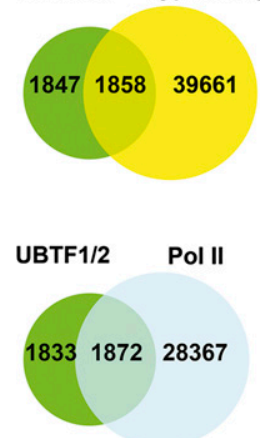

B

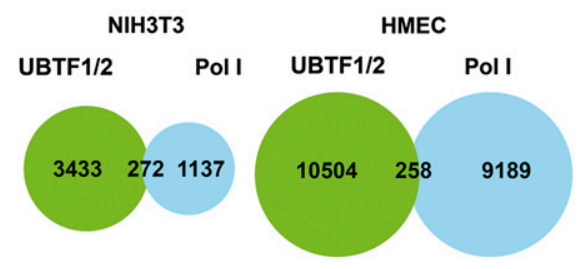

C
UBTF1/2 ChIP-seq

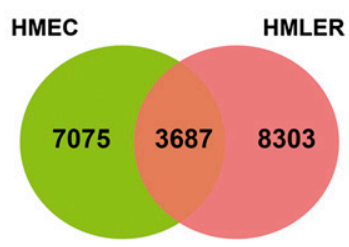

Figure 2. UBTF1/2 binding correlates with markers of open active chromatin. (A) Venn diagram indicating overlap of UBTF1/2 binding sites with various chromatin marks, DNase I HS, and Pol II binding in NIH3T3. (B) Venn diagram indicating the overlap of UBTF1/2 and Pol I (POLR1A) binding sites in NIH3T3 and HMEC. (C) Venn diagram indicating the overlap of UBTF1/2 binding sites between HMEC and HMLER cell lines.

UBTF1/2 in normal and transformed cells. Gene ontology analysis of the UBTF1/2-bound genomic regions unique to the tumorigenic HMLERs revealed biological processes involved in regulating cell cycle checkpoints, DNA damage checkpoints, and DNA damage responses (Supplemental Fig. 2F). Thus, in response to oncogenic transformation, UBTF1/2 targets an additional cohort of target genes whose products include mediators of ATR/ATM-regulated DNA damage response, signal transduction by TP53, and G1 to S transition of the cell cycle (Supplemental Tables 11-13).

Identification of functional occupancy by UBTF1/2 at the histone gene clusters

To determine if UBTF1/2 binding to Pol II regulated genes has a functional effect on gene expression, we performed expression array analysis in control and Ubtf1/2 depleted NIH3T3 cells using two independent short interfering RNAs (siRNAs) (Supplemental Table 15). We intersected the ChIP-seq and microarray expression data sets to identify genes whose expression was significantly altered by Ubtf1/2 knockdown and were bound by UBTF1/2 within 500 bp of their TSSs. Gene ontology analysis, using the MetaCore pathways software (Thomson Reuters), of the biological processes that are enriched in the intersected list identified a significant overrepresentation of genes belonging to chromatin/nucleosome assembly and DNA packaging, including canonical histone genes and histone gene variants, indicating that their transcription may be directly regulated by UBTF1/2 (Table 1 ). We then chose the histone gene clusters for further functional characterization in examining UBTF1/2's role in mediating Pol II transcription.

The five classes of genes encoding the canonical histone proteins (H2A, H2B, H3, H4, and $\mathrm{H} 1)$ are arranged in repetitive units in one major and two smaller gene clusters (Marzluff et al. 2002) somewhat reminiscent of the rDNA repeats. Histone mRNAs are transcribed at a high rate as the cells enter $\mathrm{S}$ phase in parallel with DNA replication (Marzluff et al. 2008). In addition, there are rep-

Table 1: MetaCore ontology analysis of UBTF1/2-bound genes within $500 \mathrm{bp}$ of their TSSs that are differentially expressed following Ubtf1/2 knockdown in NIH3T3 cells

\begin{tabular}{|c|c|c|c|c|}
\hline \multicolumn{3}{|c|}{ Enrichment analysis report } & \multirow{2}{*}{\multicolumn{2}{|c|}{ Common }} \\
\hline \multicolumn{3}{|c|}{ Enrichment by GO processes } & & \\
\hline \# & Processes & Total & $P$-value & In data \\
\hline 1 & Chromatin assembly & 130 & $3.185 \times 10^{-13}$ & 14 \\
\hline 2 & Enzyme-linked receptor protein signaling pathway & 820 & $3.217 \times 10^{-13}$ & 29 \\
\hline 3 & DNA packaging & 176 & $1.397 \times 10^{-12}$ & 15 \\
\hline 4 & Chromatin assembly or disassembly & 146 & $1.599 \times 10^{-12}$ & 14 \\
\hline 5 & Protein-DNA complex assembly & 146 & $1.599 \times 10^{-12}$ & 14 \\
\hline 6 & Nucleosome assembly & 124 & $3.266 \times 10^{-12}$ & 13 \\
\hline 7 & Protein-DNA complex subunit organization & 154 & $3.332 \times 10^{-12}$ & 14 \\
\hline 8 & System development & 3997 & $4.532 \times 10^{-12}$ & 64 \\
\hline 9 & Multicellular organismal development & 4704 & $7.630 \times 10^{-12}$ & 70 \\
\hline 10 & DNA conformation changes & 205 & $1.270 \times 10^{-11}$ & 15 \\
\hline
\end{tabular}

Only the most significantly enriched gene ontologies are listed. $P$-value denotes the significance of the number of genes from the intersected list (In data) when compared to the total number of genes in the gene ontology classification (Total).

\section{Genome Research}


lication-independent variant histone genes that are not located within the histone gene clusters and are constitutively transcribed throughout the cell cycle. These genes encode histone variants such as H1.0, H2A.Z, H2A.X, H3.3A, and H3.3B, which are known to have distinct functions (Marzluff et al. 2008).
We detected robust peaks of UBTF1/2 enrichment at 33/45 canonical histone genes and $4 / 6$ histone $\mathrm{H} 1$ genes within histone cluster 1, 4/10 genes within histone cluster 2 (Fig. 3A,B), and also at the single histone $\mathrm{H} 4$ gene located on chromosome 12 in NIH3T3, but none at the small histone cluster 3 . In addition, UBTF1/2

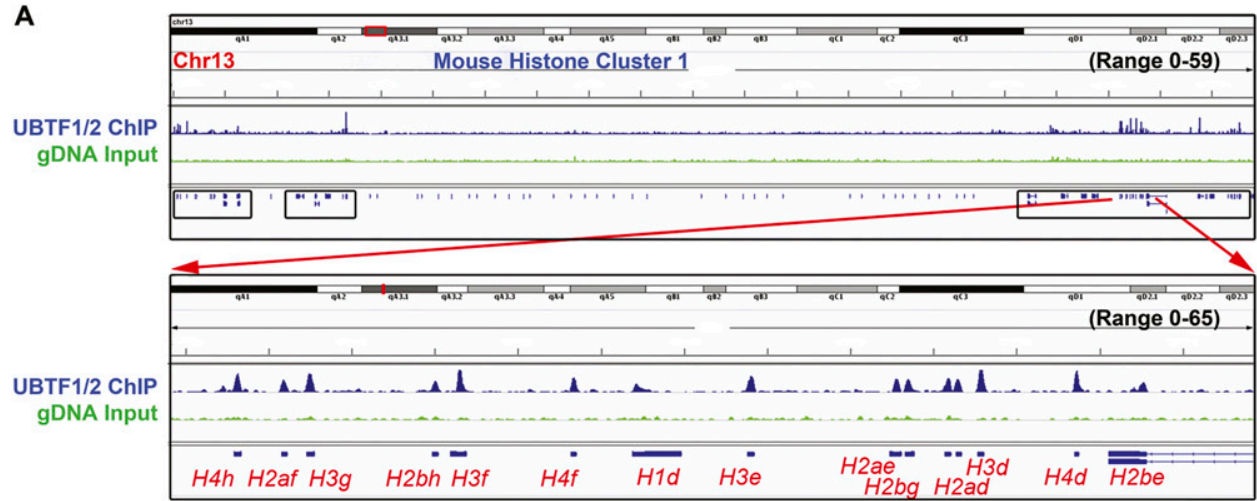

B

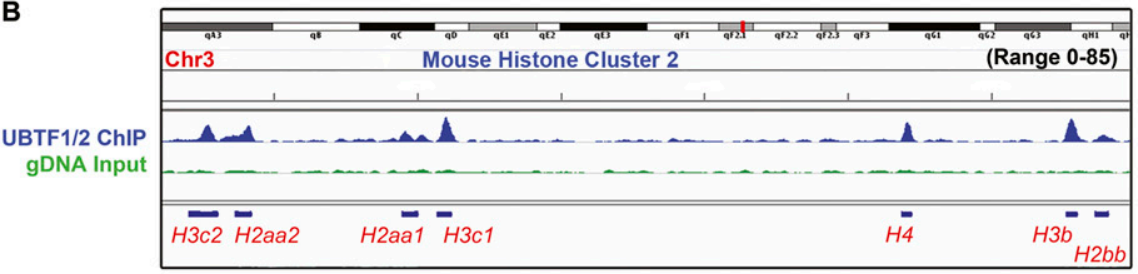

C

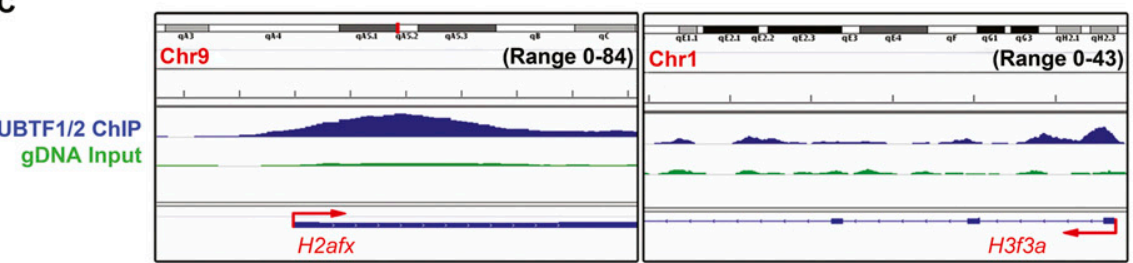

D

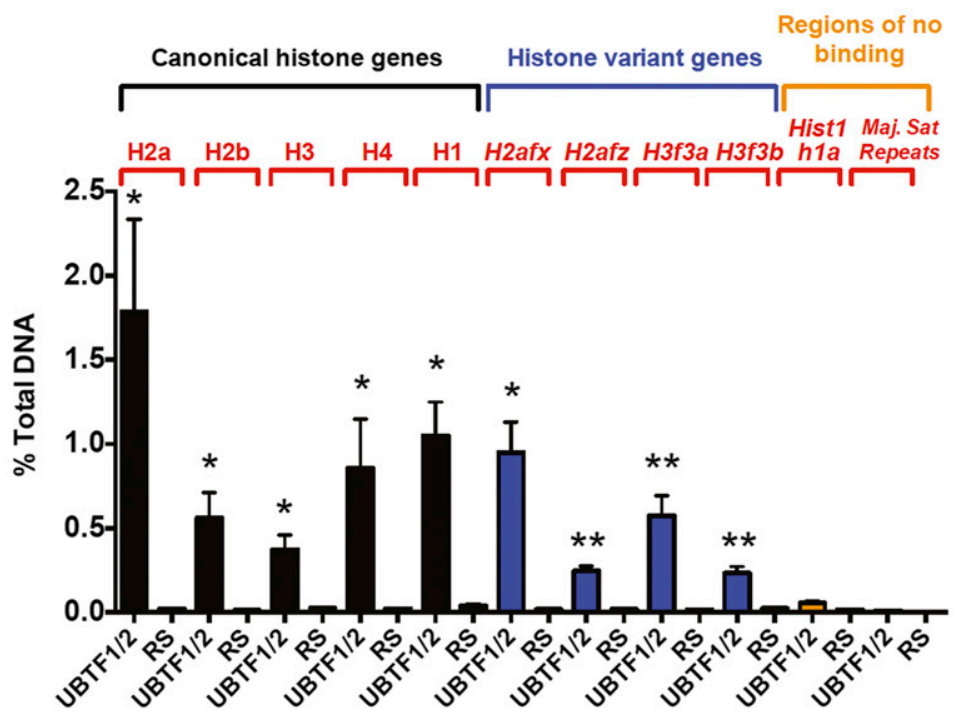

Figure 3. UBTF1/2 binds histone genes. ( $A-C$ ) IGV (Integrated Genome Viewer) screenshots of mapped reads from UBTF1/2 ChIP and input gDNA at mouse histone gene cluster $1(A)$, cluster $2(B)$, and histone variant genes $H 2 a f x$ and $H 3 f 3 a(C)$ in NIH3T3 cells. (D) qChIP analysis of UBTF1/2 binding to canonical and variant histone genes in NIH3T3 cells. qChIPs were performed as described in Figure 1D $\left(n=4 ;\right.$ Ave \pm SEM). $\left({ }^{*}\right) P$-value $<0.05$, $(* *) P$-value $<$ 0.01 , compared to corresponding RS samples. Amplicons at Hist $1 \mathrm{~h} 1 \mathrm{a}$ and major satellite repeats were used as a negative control for UBTF1/2 binding. 
binding was detected at six histone gene variants, which are dispersed across the genome, including H2afy, H2afx, H2afz, H3f3a, $H 3 f 3 b$, and $H 1 f O$ (Fig. 3C). A similar enrichment of UBTF1/2 was observed at histone gene clusters and histone variants in HMEC (Supplemental Fig. 3B,C; Supplemental Tables 16, 17).

Using qChIP, we confirmed significant enrichment of UBTF1/2 binding at the canonical and histone variant genes compared to two regions that ChIP-seq data indicate are not bound by UBTF1/2 (the major satellite repeats and an amplicon specific to Hist1h1a) (Fig 3D). These data suggest that histone genes are likely bona fide targets of UBTF1/2.

\section{UBTF1/ 2 mediates histone gene expression}

As Ubtf1/2 depleted NIH3T3 cells display a delay in G1-S progression (Supplemental Fig. 3D), we examined the effect of $U b t f 1 / 2$ depletion on histone mRNA levels in sorted $\mathrm{G} 1$ and $\mathrm{S}$ populations from $\operatorname{sir} E g f p$ control and $U b t f 1 / 2$ knockdown samples to control for any cell cycle bias. Histone $\mathrm{H} 4$ and H2afx mRNA levels were significantly reduced following $U b t f 1 / 2$ knockdown in the G0/G1 and in the $\mathrm{S}$ phase samples (Fig. 4A), suggesting that the decrease in histone mRNA expression is a direct consequence of $U b t f 1 / 2$ knockdown rather than accumulation of cells in G1. Indeed, in the G0/G1 populations, mRNA levels of all canonical and histone variant mRNAs were significantly decreased following Ubtf1/2 depletion (Fig. 4B). Thus, UBTF1/2 plays a permissive role in mediating mRNA expression of canonical and variant histone genes rather than functioning in the activation of canonical histone gene expression in S phase. Furthermore, consistent with UBTF1/2 regulating mRNA expression of histone genes, transient $U b t f 1 / 2$ knockdown significantly reduced the abundance of histone proteins as represented by H2A and H1 (Supplemental Fig. 4A,B).

\section{UBTF1/2 mediates Pol II recruitment and transcription of histone genes independent of chromatin modifications or nucleosome loading}

To examine the mechanism underlying UBTF1/2-mediated regulation of histone gene expression, we performed sequential ChIP assays (ChIP-reChIP) experiments to investigate the overlap between UBTF1/2 and Pol II or Pol I at histone genes. The data showed that UBTF1/2 and Pol II simultaneously occupy histone genes (Supplemental Fig. 4C). Consistent with the lack of overlap observed in UBTF $1 / 2$ and Pol I binding across the genome (Fig. 2B), we observed no enrichment in Pol I occupancy at histone genes (Fig. 4C; Supplemental Fig. 4C). This eliminates the possibility of UBTF1/2 recruiting Pol I to histone gene loci.

To confirm that UBTF1/2 directly regulates transcription of histone genes via Pol II, we examined Pol II recruitment to histone genes in response to modulation of UBTF1/2 levels. To a large extent, the phosphorylation status of the C-terminal domain (CTD) of the largest subunit of Pol II defines the stage of transcription (Phatnani and Greenleaf 2006). Pol II is recruited into the PIC with a hypophosphorylated CTD; the CTD is phosphorylated on Serine 5 (Ser5) during initiation and promoter clearance and then on Serine 2 (Ser2) during elongation (Fuda et al. 2009). We observed significant reductions in total Pol II and Pol II (Ser5) loading at histone $\mathrm{H} 2 \mathrm{a}, \mathrm{H} 4$, and $\mathrm{H} 1$ genes in $U b t f 1 / 2$ depleted cells (Fig. 4C), suggesting that UBTF1/2 is required for recruitment and initiation of Pol II transcription at histone genes. Interestingly, we also observed proportionally greater reduced occupancy of elongating Pol II (Ser2) at histone genes compared to the observed reduction in
Pol II loading/initiation following Ubtf1/2 knockdown. This suggests a role for UBTF1/2 in mediating Pol II elongation rates, consistent with its ability to mediate Pol I elongation (Stefanovsky et al. 2006).

Ubtf1/2 knockdown did not correlate with any significant changes in chromatin modifications at histone $\mathrm{H} 2 \mathrm{a}$ and $\mathrm{H} 1$ genes (Supplemental Fig. 4D), indicating that UBTF1/2's role in regulating histone gene expression is downstream or independent of the establishment of the euchromatic environment within the histone gene clusters. Furthermore, we did not observe significant changes in the enrichment of histones $\mathrm{H} 3, \mathrm{H} 4$, or linker histone $\mathrm{H} 1$ loading at histone $\mathrm{H} 2 \mathrm{a}$ and $\mathrm{H} 1$ genes following $U b t f 1 / 2$ knockdown (Supplemental Fig. 4D). Thus, UBTF1/2 does not alter nucleosome loading at histone genes. To further examine the effect of UBTF1/2 binding on chromatin remodeling events at histone genes, we designed primers tiling the 340-bp region that spans the promoter, the TSS, and the transcribed region of Hist1h2ad and performed qChIP (Fig. 4D). UBTF1/2 binding at Hist1h2ad was $\sim 10$ - to 15 -fold higher at amplicons within the transcribed region compared to the promoter (Fig. 4E). We then examined nucleosome positioning at Hist1h2ad by chromatin accessibility assay (CHART-PCR) (Rao et al. 2001). Micrococcal nuclease (MNase) accessibility measurements of control samples revealed the region spanning -194 to +15 bp of Hist 1 h2ad (amplicons P-1, P-2, and A) is highly sensitive to MNase digestion compared to the transcribed region ( +32 to $+144 \mathrm{bp}$, amplicons B and C) (Fig. 4F), suggesting that it is depleted of a nucleosome as expected for a highly constitutively transcribed gene. Despite the fact that histone genes are present within an open chromatin context (Hovhannisyan et al. 2003; Medina et al. 2012), Ubtf1/2 knockdown led to a significant decrease in protection against MNase digestion at the promoter and also at the transcribed region (Fig. 4F). This suggests that UBTF1/2 interacts directly with DNA as opposed to nucleosomes and its binding modulates DNA accessibility to promote Pol II binding independent of nucleosome occupancy. The data further suggest that UBTF1/2wrapped chromatin (Stefanovsky et al. 2001) is a preferred template for Pol II rather than histone-wrapped chromatin.

\section{UBTF2 is required for optimal histone gene expression}

UBTF2 is inactive in regulating rDNA transcription compared with UBTF1 (Grummt 1999; Hannan et al. 1999; Stefanovsky and Moss 2008) and does not function in remodeling chromatin at the rDNA repeats in the absence of UBTF1 (Sanij et al. 2008). To address the relative contribution of UBTF1 and UBTF2 isoforms to Pol II histone gene expression, we performed replacement experiments with rat FLAG-Ubtf1 and FLAG-Ubtf2 cDNAs, which contain nucleic acid sequence differences in the region targeted by the murine-specific sirUbtf1/2 (Supplemental Fig. 5A; Sanij et al. 2008), and performed qChIP assays using anti-FLAG antibodies. We found that both FLAG-rUBTF1 and FLAG-rUBTF2 can interact with histone genes independently of endogenous UBTF1 and UBTF2 (Supplemental Fig. 5B). Next, we performed RNAi knockdown targeting Ubtf1 and Ubtf2 mRNAs or only Ubtf1 mRNA (we are unable to selectively target Ubtf2 by RNAi) (Fig. 5A). While total UBTF1/2 enrichment was significantly reduced at histone genes in Ubtf1/2 depleted cells, Ubtf1 knockdown cells exhibited similar enrichment of total UBTF1/2 loading to control cells, indicating that UBTF2 is able to fully occupy histone genes independently of UBTF1 (Fig. 5B). Moreover, while Ubtf1/2 knockdown significantly reduced histone $\mathrm{H} 2 \mathrm{a}, \mathrm{H} 1$, and $\mathrm{H} 4 \mathrm{mRNA}$ levels, Ubtf1 knockdown failed to reduce histone mRNA levels, demonstrating that UBTF2 is 
A

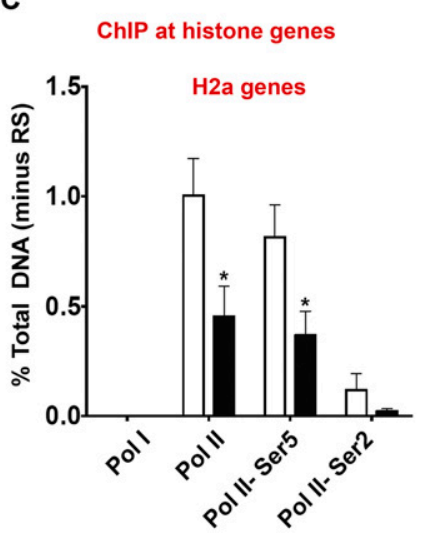

D

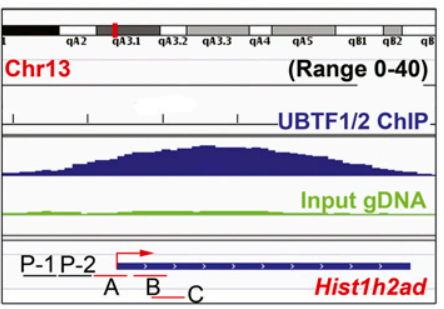

C $\square$ sirEgfp

sirUbtf1/2

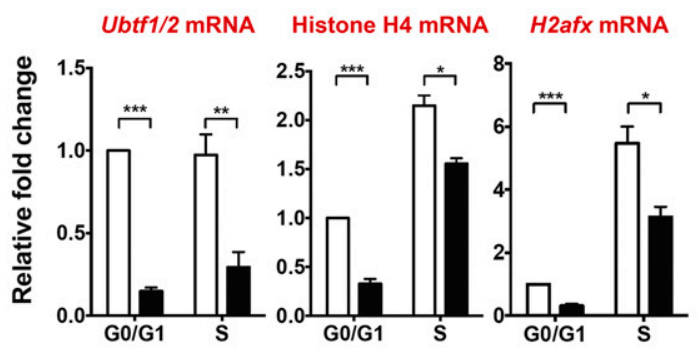

E

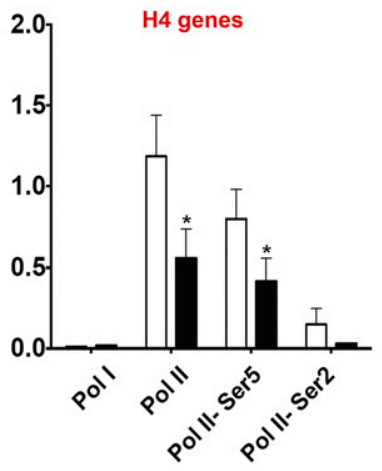

UBTF1/2 ChIP

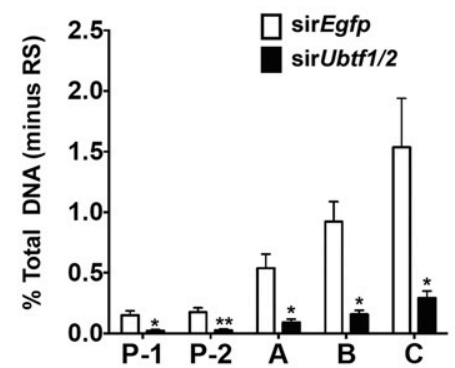

B

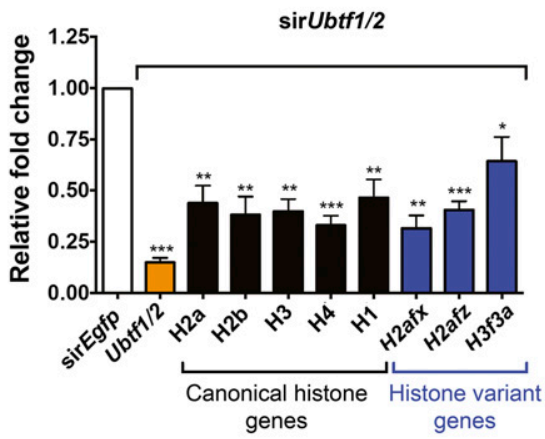

mRNA expression in G1

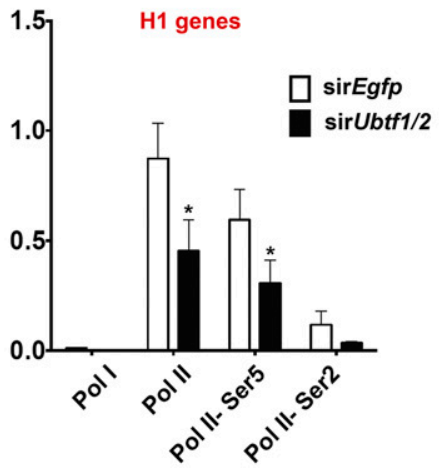

F

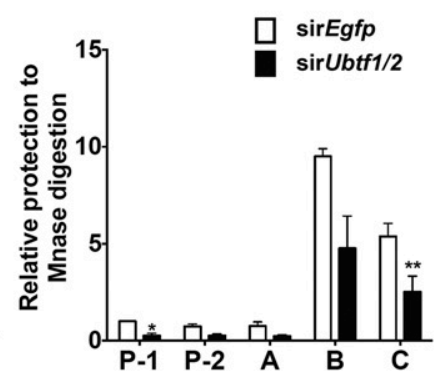

Figure 4. UBTF1/2 regulates histone gene expression by mediating Pol II recruitment. (A) NIH3T3 cells were transfected with sirEgfp or sirUbtf1/2\#1 for $48 \mathrm{~h}$, then collected and incubated with Vybrant DyeCycle Violet stain (Life Technologies). Cells were then analyzed using BD FACSAria, and G1 and S phase populations were sorted based on DNA content. Total RNA was extracted and Ubtf1/2, Histone H4, and H2afx mRNA levels were determined by reverse transcription qPCR. mRNA levels were normalized to beta-2-microglobulin (B2m) mRNA and expressed as fold change relative to sirEgfp/G0/G1 $(n=4)$. (B) Total RNA samples from G1 population of NIH3T3 cells transfected with sirEgfp or sirUbtf1/2\#1 as in $A$ were analyzed by reverse transcription qPCR for mRNA expression of various canonical and variant histone genes as indicated $(n=4)$. (C) UBTF1/2 mediates Pol II recruitment, initiation, and elongation at histone genes. qChIP analysis of the histone $\mathrm{H} 2 \mathrm{a}, \mathrm{H} 4$, and $\mathrm{H} 1$ genes in sirEgfp- or sirUbtf1/2\#1-transfected NIH3T3 cells using antibodies against Pol I $(n=2)$, total Pol II $(n=7)$, phospho Pol II-Ser5 $(n=4)$, or phospho Pol II-Ser2 $(n=4)$. The percentage of DNA immunoprecipitated with the indicated antibodies or RS was calculated relative to the unprecipitated input control. Percentage of DNA of RS controls was subtracted. (D) Ubtf1/2 knockdown leads to increased DNA accessibility at Hist1 h2ad. Screenshots of IGV with the mapped reads from UBTF1/2 ChIP and input gDNA in NIH3T3 to Hist1 h2ad. Primers used for qPCR are indicated (Supplemental Table 21). (E) qChIP analysis of UBTF1/2 binding at Hist1h2ad in sirEgfp or sirUbtf1/2\#1 transfected NIH3T3 cells. qChIPs were performed as described in Figure 4C $(n=3)$. ( $F)$ Chromatin remodeling of the Hist1h2ad by UBTF1/2. Nuclei from $\mathrm{NIH} 3 \mathrm{~T} 3$ cells transfected with either sirEgfp or sirUbtf1/2\#1 for $48 \mathrm{~h}$ were incubated with or without MNase. Extracted gDNA was subjected to qPCR using primers outlined in $D$. MNase accessibility was expressed as a percentage of undigested gDNA samples $(n=3)$. In all graphs $(A-F)$, error bars represent Ave \pm SEM, $\left(^{*}\right) P$-value $<0.05,\left({ }^{* *}\right) P$-value $<0.01,\left({ }^{* *}\right) P$-value $<0.001$, compared to corresponding sirEgfp samples.

sufficient to regulate histone gene expression in the absence of UBTF1 (Fig. 5B). In direct contrast, at the rDNA loci both Ubtf1/2 and $U b t f 1$ knockdown led to a similar 50\% reduction in $45 \mathrm{~S}$ rRNA levels (Fig. 5A). Thus, although UBTF2 is known to be inactive in Pol I transcription (Grummt 1999; Hannan et al. 1999; Stefanovsky and Moss 2008) and chromatin remodeling at rDNA (Sanij et al. 
A

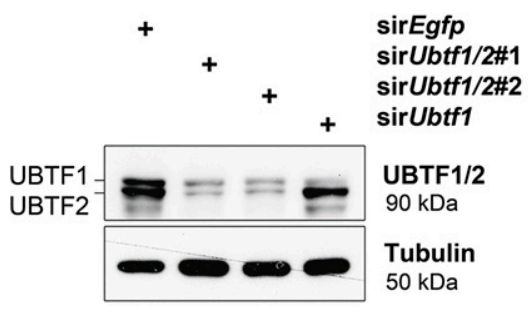

45S rRNA

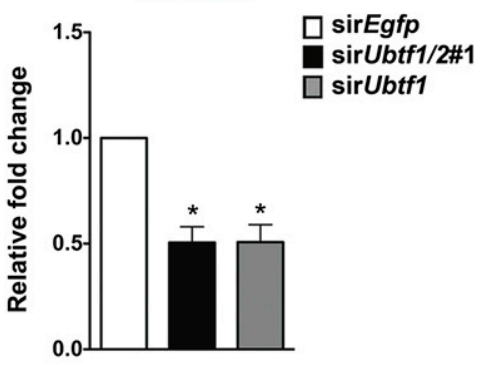

B

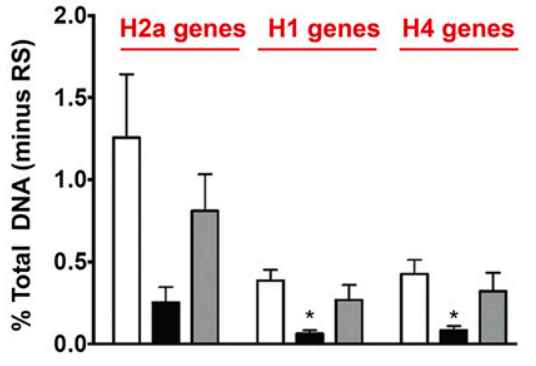

Histone mRNA expression

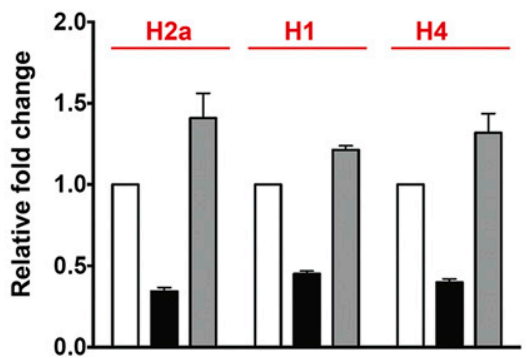

Figure 5. The UBTF2 isoform mediates histone gene expression. (A) Total protein lysates from NIH3T3 cells transfected with sirEgfp, sirUbtf1/2\#1, sirUbtf1/2\#2, or sirUbtf1 for $48 \mathrm{~h}$ were analyzed by Western blotting (top panel). RNA was also extracted and $45 \mathrm{~S}$ rRNA precursor levels were determined by reverse transcription QPCR using primers to the $5^{\prime}$ external transcribed region (ETS) (Supplemental Table 20). 45S rRNA levels were normalized to Gapdh mRNA and expressed as fold change relative to sirEgfp control $\left(n=3\right.$; Ave \pm SEM). ( $\left.{ }^{*}\right)$ P-value $<0.05$ (bottom panel). (B) (Top panel) qChIP analysis of UBTF1/2 binding to histone $\mathrm{H} 2 \mathrm{a}, \mathrm{H} 1$, and $\mathrm{H} 4$ genes in NIH3T3 cells transfected with either sirEgfp, sirUbtf1/2\#1, or sirUbtf1 for $48 \mathrm{~h}\left(n=3\right.$; Ave \pm SEM). ( $\left.{ }^{*}\right)$ P-value $<0.05$ compared to sirEgfp sample. qChIPs were performed as described in Figure 4C. (Bottom panel) G1 populations of NIH3T3 cells transfected as above were sorted as described in Figure $4 \mathrm{~A}$, and total RNA was extracted. Histone $\mathrm{H} 2 \mathrm{a}, \mathrm{H} 1$, and $\mathrm{H} 4$ mRNA levels were determined by reverse transcription qPCR. mRNA levels were normalized to $B 2 m$ mRNA and expressed as fold change relative to the sirEgfp control $\left(n=4\right.$; Ave \pm SEM). $\left(^{*}\right) P$-value $<0.05$ relative to corresponding sirEgfp samples.

2008), these data demonstrate that UBTF2 functions to enhance Pol II gene expression.

\section{Ubtf1/2 knockdown leads to DNA damage and genomic instability}

In eukaryotes, DNA damage and repair occurs in the context of chromatin and thus histone availability affects genome integrity and stability (Ye et al. 2003; Gunjan et al. 2005). Thus, the loss of histone gene expression following Ubtf1/2 depletion might be associated with DNA damage and/or genome instability. To investigate this possibility, we used the comet assay to detect DNA damage at the single-cell level and found that Ubtf1/2 knockdown cells exhibited significantly longer comet tails than those of control cells, indicating that they accumulated DNA damage (Fig. 6A).

The appearance of micronuclei is another characteristic marker of damaged chromosomes. Micronuclei are small nuclei separated from the main nucleus, containing chromosomes or chromosome fragments derived from mitotic spindle dysfunction (Fenech 2006). We performed a cytokinesis-block micronucleus $(\mathrm{CBMN})$ assay to quantify micronuclei appearing after a single complete nuclear division. Ubtf1/2 knockdown cells displayed
(Supplemental Fig. 6) and do not merely
reflect genomic instability of the rDNA repeats but rather genome-wide chromosome instability.

\section{Discussion}

\section{UBTF1/ 2 couples the regulation of a subset of highly transcribed Pol I and Pol II genes}

In this study we demonstrate a fundamental role for the Pol I transcription factor UBTF1/2 in regulating highly transcribed Pol II genes, including the histone gene clusters. Moreover, in transformed cells, we demonstrate that UBTF1/2 is enriched at an additional cohort of genes involved in DNA damage and repair, including mediators of ATR/ATM-regulated DNA damage response, signal transduction by TP53, and G1 to S transition of the cell cycle. Activation of the ATM/ ATR-regulated DNA damage network following oncogenic stress is proposed to be a biological response to delay or protect against cancer (Bartkova et al. 2005) by pausing cells in S phase or G2 to allow appropriate repair of damaged DNA. It is tempting to speculate that this additional cohort of UBTF1/2 target genes represents a regulated response to oncogenic stress to couple the rate of rDNA transcription with DNA integrity and fidelity of chromatin assembly.

Thus collectively, the data indicate that UBTF1/2 binding is dynamic, context dependent, and potentially associated with

\section{Genome Research} www.genome.org 
A

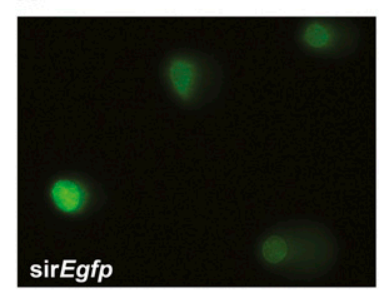

B

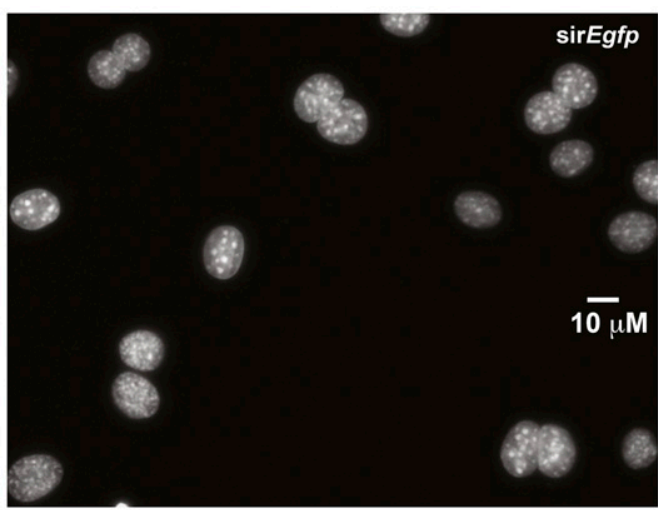

C

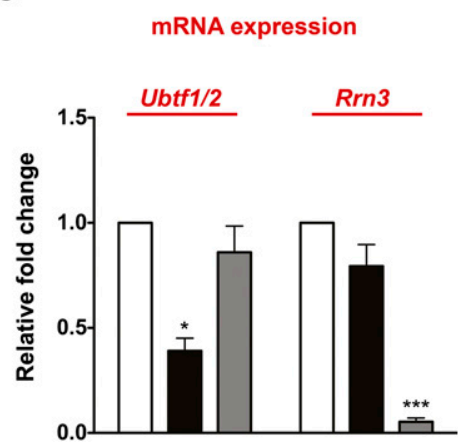

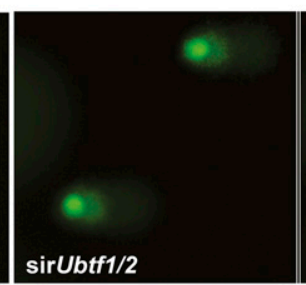

$100 \mu \mathrm{M}$
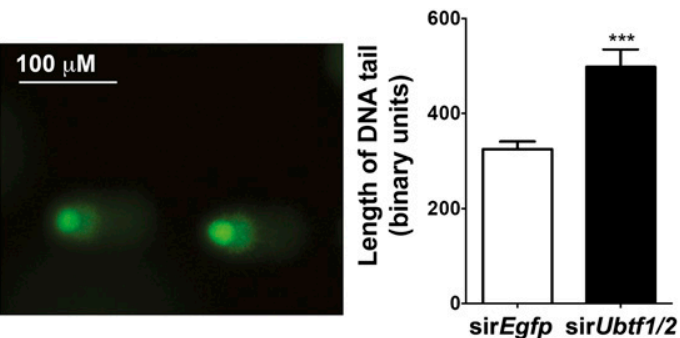

sirEgfp sirUbtf1/2

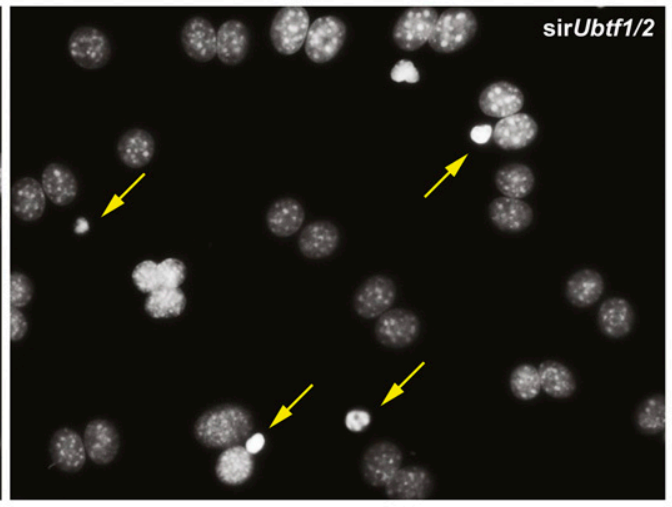

D

45S rRNA expression

Micronucleus assay
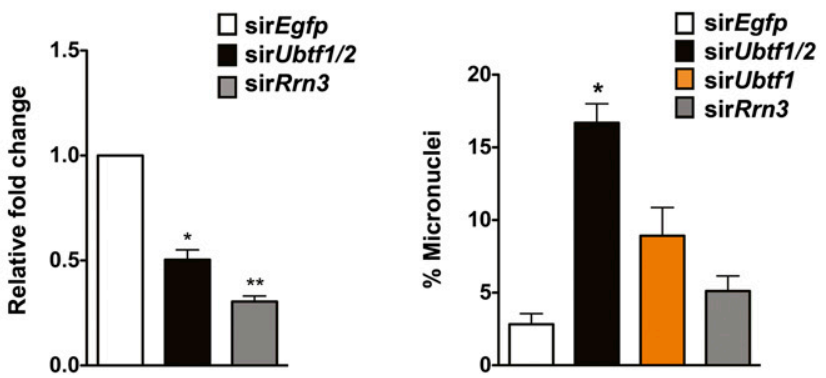

Figure 6. Ubtf1/2 knockdown leads to DNA damage and genomic instability. $(A)$ NIH3T3 cells were transfected with sirEgfp or sirUbtf1/2\#1 for $48 \mathrm{~h}$ and DNA damage was measured by comet assay. Representative images of SYBR green-stained DNA of sirEgfp control cells, showing undamaged and supercoiled DNA remaining within the nuclear membrane, while in sirUbtf1/2\#1 cells, denatured DNA fragments migrate out from the nucleus in a comet tail. The tail length for $\sim 50$ nuclei for each sample from two independent experiments was measured using metamorph software. The graph on the right panel represents Ave \pm SEM. (*) P-value $<0.05$ compared to sirEgfp control. (B) Ubtf1/2 knockdown leads to abnormal mitosis as measured by the CBMN assay. NIH3T3 cells transfected with sirEgfp or sirUbtf1/2\#1 were incubated for $24 \mathrm{~h}$, then Cytochalasin B, an inhibitor of cytokinesis, was added at $3 \mu \mathrm{g} / \mathrm{mL}$ for a further $24 \mathrm{~h}$. DAPI staining was then performed and percentages of binucleated cells exhibiting micronuclei were scored (yellow arrows) $(n=3 ;$ Ave \pm SEM). $(*) P<0.05$. (C) Forty-eight hours after transfecting NIH3T3 cells with sirEgfp, sirUbtf1/2\#1, or sirRrn3, total RNA was extracted, and Ubtf1/2 mRNA, Rrn3 mRNA, and 45S rRNA precursor levels were determined by reverse transcription qPCR. mRNA levels were normalized to $B 2 m$ mRNA and expressed as fold change relative to the sirEgfp control $\left(n=3\right.$; Ave \pm SEM). $\left(^{*}\right) P$-value $<0.05,\left({ }^{* *}\right) P$-value $<0.01,\left({ }^{* * *}\right) P$-value $<0.001$, compared to corresponding sirEgfp controls. ( $\left.D\right)$ NIH3T3 cells were transfected with siRNA oligos as indicated, and the CBMN assay was performed as described in $B(n=3)$. Approximately 100 cells were counted in each experiment and percentages of binucleated cells exhibiting micronuclei were scored (Ave \pm SEM). ( $\left.{ }^{*}\right) P$-value $<0.05$ compared to sirEgfp control.

malignant transformation. While a number of Pol II transcription factors have been implicated in the regulation of Pol I, for example, MYC, TP53, and RUNX2 (Hannan et al. 2013), our data provide the first specific in vivo example of a Pol I factor with dual functions in the regulation of Pol I and Pol II.

Interestingly, although ribosomal protein (RP) encoding genes, like histone genes, are also highly expressed genes (CastilloDavis et al. 2002), they did not appear in the gene ontological analysis of genes bound by UBTF1/2 in NIH3T3 (Supplemental Fig. 2A) nor did they change in mRNA levels upon Ubtf1/2 knockdown (Supplemental Table 15). In fact, UBTF1/2 ChIP-seq peaks in NIH3T3 cells were detected at only five RP genes. Thus, the data do not support a model in which ribosome biogenesis is coordinated at the transcriptional level by UBTF1/2. Instead, it is consistent with the prevailing view that in mammals, the major mode of coordinated regulation of ribosome biogenesis is achieved post-transcriptionally via specific conserved signaling networks including the PI3K/AKT/TOR and ERK pathways (White 2008; Chan et al. 2011; Hannan et al. 2011, 2013; Bywater et al. 2013; Chauvin et al. 2014). This appears in contrast to lower organisms such as yeast where transcription of the rRNAs by Pol I and the RPs by Pol II is coordinated (Laferte et al. 2006; Berger et al. 2007). We note that in higher eukaryotes, RPs have evolved extra-ribosomal functions, for example, in regulating nucleolar stress and TP53 
accumulation through the binding of certain RPs to MDM2 (Boulon et al. 2010; Deisenroth and Zhang 2010; Hein et al. 2013). The transcriptional uncoupling of rRNA and RP synthesis in higher eukaryotes may allow for regulation of the extra-ribosomal functions of RPs by mechanisms and signaling pathways independent of those that modulate ribosome biogenesis.

Ubtf1/2 are splice variants from a single transcript and their expression is dynamically regulated by $>90 \%$ during terminal differentiation and proliferation, which coincides with dramatic changes in the rDNA transcription rate (Larson et al. 1993; Moss and Stefanovsky 2002; Sanij et al. 2008; Sanij and Hannan 2009; Poortinga et al. 2011, 2014; Bywater et al. 2012). Conversely, they are robustly up-regulated under proliferative conditions (Moss and Stefanovsky 2002; Sanij and Hannan 2009; Bywater et al. 2012; Poortinga et al. 2014). Critically, our previous studies demonstrated that UBTF2 does not function in Pol I transcription, raising the perplexing question about the UBTF2 function in vivo (Sanij et al. 2008). In this study we demonstrated that UBTF2 is sufficient in the absence of UBTF1 to modulate Pol II gene expression. Moreover, depletion of Ubtf1 alone had little effect on Pol IIdependent transcription of the histone genes but robustly repressed Pol I-dependent transcription of the rDNA (Fig. 5). Together, these data suggest that UBTF1 functions predominantly in regulating Pol I, while UBTF2 functions in regulating Pol II. Alternative splicing for the same gene to yield two distinct mRNA isoforms, with specific roles in regulating Pol I (Ubtf1) and Pol II (Ubtf2) transcription, respectively, appears to be an ideal evolutionary mechanism to couple the regulation of highly transcribed genes required for fundamental processes such as rRNA synthesis and chromatin assembly.

\section{Recruitment of UBTF1/ 2 to open chromatin}

The interphase genome is organized into self-interacting topological domains flanked by boundaries. These regions are remodeled by Pol II and topoisomerase activities creating supercoiling domains. Underwound, negatively supercoiled domains are transcriptionally active, enriched in "open" chromatin fibers, DNase I hypersensitive sites, and TSSs (Naughton et al. 2013) reminiscent of chromatin structures bound by UBTF1/2 (Figs. 1C,F, 2A). Like other HMG proteins, UBTF1/2 has little intrinsic DNA binding specificity in vitro. One possibility is that UBTF1/2 may recognize specific topological features that are characteristic of specific DNA regions with very high constitutive transcriptional activity. Indeed, intersection of UBTF1/2-bound regions with DNA supercoiling data across seven genomic loci that distinguish between negative supercoiled "underwound" and positively supercoiled "overwound" DNA (Naughton et al. 2013) demonstrates that $\sim 83 \%$ of the overlapping regions represent underwound DNA (data not shown). Thus, UBTF1/2 may "mark" and stabilize regions of constitutively active "underwound" DNA structures. In turn, this may facilitate rapid induction of mRNA synthesis during the cell cycle or in response to extracellular stimuli.

\section{UBTF1/ 2 as a mediator of genomic stability}

UBTF1/2 deficiency, but not reductions in Pol I transcription per se, resulted in genome-wide instability in NIH3T3 cells (Fig. 6). Almost certainly the role UBTF1/2 plays in maintaining high levels of canonical and noncanonical histones and other genes involved in chromatin assembly and DNA damage response contributes to the genomic instability upon Ubtf1/2 knockdown. This would be consistent with the observations that histone insufficiency during $\mathrm{S}$ phase results in spontaneous DNA damage and genomic instability (Han et al. 1987; Myung et al. 2003; Ye et al. 2003; Prado and Aguilera 2005; Clemente-Ruiz and Prado 2009) and that manipulation of core histone function or abundance causes defects in chromosome segregation (Meeks-Wagner and Hartwell 1986; Pinto and Winston 2000).

Thus, through its ability to coordinately regulate the expression of some of the most highly transcribed Pol I and Pol II genes in the genome including the histones clusters and rDNA repeats, our data demonstrate a previously unrecognized but fundamental role for the two isoforms of UBTF1/2 in coupling Pol I transcription and the cell's capacity to grow with the fidelity of chromatin assembly and DNA repair. Intriguingly, it has recently been demonstrated that inhibition of Pol I is an effective mechanism to treat hematological cancers with drugs targeting Pol I transcription now in clinical trials (Bywater et al. 2012 , 2013). We propose that the unique characteristics of UBTF1/2 to target a subset of Pol II genes critical for cell proliferation, in addition to Pol I, make it a highly attractive target for cancer therapy.

\section{Methods}

\section{ChIP and ChIP-seq analysis}

Standard ChIP was carried out as described (Poortinga et al. 2004; Sanij et al. 2008). Antibodies and primer sequences are listed in the Supplemental Data. ChIP-reChIP experiments were carried out as described in Mendoza-Parra (2012). Anti-UBTF1/2 sera were crosslinked to protein A beads using disuccinimidyl suberate. UBTF1/2 ChIP products were eluted in 1\%SDS, $0.1 \mathrm{M} \mathrm{NaHCO}_{3}$, diluted 1:10 and subjected to a second ChIP using antibodies directed against Pol I, Pol II, or RS.

For ChIP-seq of UBTF1/2 ChIP DNA and input gDNA from NIH3T3, libraries from two biological replicates were prepared for the Illumina Genome Analyzer II platform at Peter MacCallum Cancer Center. ChIP-seq libraries of histone modifications and Pol II in NIH3T3 libraries were also prepared for the Illumina Genome Analyzer II platform. Sequencing reads were mapped to the mouse genome database mm9 using the Burrows-Wheeler Aligner (BWA) (Li and Durbin 2009). ChIP-seq of UBTF1/2 ChIP, Pol I, and gDNA samples in HMEC was performed using the Illumina Genome Analyzer II platform, while ChIP-seq of UBTF1/2 ChIP and gDNA samples in HMLER cells was performed using Illumina HiSeq 1000. Sequences were mapped to the human genome (hg19). MACS 1.4 (Model-based Analysis of ChIP-seq [Zhang et al. 2008]) was used to call peaks over input DNA, and the fold enrichment for each peak was calculated using the R package (Gentleman et al. 2004). Only peak regions with a false discovery rate (FDR) $<10 \%$ and $P$-value $<$ 0.00001 were selected and annotated with RefSeq annotation using the R package ChIPpeakAnno (Zhu et al. 2009). The distribution of UBTF1/2 peaks relative to RefSeq genes as well as the comparison of ChIP-seq data sets was performed using the SoleSearch software tool (Blahnik et al. 2010). The DNase I hypersensitivity profile in NIH3T3 cells was obtained from the ENCODE UCSC Genome Browser (https://www.encodeproject.org/, Accession: ENCSR000CNS).

\section{Gene expression analysis}

We performed microarray analysis (Affymetrix, Mouse Exon ST 1.0 arrays) on three biological replicates of $U b t f 1 / 2$ knockdown samples using two independent siRNA oligos, a nonsilencing sirEgfp or mock-transfected NIH3T3 samples (Supplemental Table 18). The arrays were normalized using the robust multiarray average expression measure (RMA) (Irizarry et al. 2003), and differential expression was then determined using a linear model and the Limma package (http://bioinf.wehi.edu.au/limma/) (Smyth 2005). Moderated $t$-statistics were generated and signifi-

\section{Genome Research}


cance was assessed using the log-fold change and FDR-adjusted $P$-value.

\section{Correlation analysis of UBTF1/2 ChIP-seq and microarray expression data}

Expression data of exponentially growing NIH3T3 were obtained using Affymetrix, Mouse Exon ST 1.0 arrays (mock samples from previous section). Triplicates were normalized using the RMA method (Irizarry et al. 2003) and expression values for all genes, genes with significant UBTF1/2 peaks $<2 \mathrm{~kb}$ from their TSS, or genes with no UBTF1/2 binding at their TSS, were obtained. Statistical significance between the groups was assessed using $t$-tests.

\section{Cell culture, siRNA knockdown, and RNA expression analysis}

NIH3T3 cells were cultured in Dulbecco's modified Eagle's medium (DMEM) with $10 \%$ fetal bovine serum (FBS) at $37^{\circ} \mathrm{C}$. HMECs and HMLER cells were cultured in HuMEC ready medium (12752010, Life Technologies). Dharmafect 2 reagent (Dharmacon) was used to transfect siRNA at $40 \mathrm{nM}$. The tetracycline-regulated rat Ubtf1 and Ubtf2 MEF-3T3 cell lines were established as reported in Sanij et al. (2008). Cells were lysed, RNA was extracted, and first-strand cDNA was synthesized using random hexamer primers and Superscript III (Invitrogen). Quantitative PCR (qPCR) was performed in duplicate using the FAST SYBR Green dye on the StepOnePlus realtime PCR system (Applied Biosystems). Primer sequences are listed in (Supplemental Table 20).

\section{Western blotting and immunofluorescence}

Twenty to fifty milligrams of whole-cell lysates were resolved by SDSPAGE, electrophoretically transferred onto PVDF membranes (Millipore), and analyzed using enhanced chemiluminescence (ECL) detection (GE Healthcare). For IF, cells were fixed in $4 \%$ paraformaldehyde (10 min at room temperature), washed with PBS, and blocked with $5 \%$ skim milk powder, $0.5 \%$ chicken serum in PBS, and $0.3 \%$ Triton X-100 for $30 \mathrm{~min}$. Cells were sequentially incubated with anti-UBTF1/2 sera and Alexa Fluor 594 anti-rabbit secondary antibody (Molecular Probes). Stained cells were fixed again with $4 \%$ formaldehyde and counterstained with DAPI in Vectashield mounting media (Vector Labs). Images were acquired on an Olympus BX-51 microscope equipped with a Spot RT camera (model 25.4), using the UPlanAPO 40×, NA 1.2 water immersion objective and the Spot Advanced software, version 4.6.4.3. Settings for adjusting the image after acquisition (i.e., gamma adjust and background subtract settings) were identical for all images. A detailed FISH protocol is provided in the Supplemental Methods.

\section{COMET assay}

Cells were collected and processed as described in the manufacturer's protocol (Trevigen, Comet Assay 4250-050-K). Images were acquired on an Olympus BX-51 microscope using the Olympus UPlanAPO $20 \times$, NA 1.2 water immersion objective as described above. Quantitation was performed using MetaMorph (Molecular Devices).

\section{CHART-PCR assay}

Cells were collected and mononucleosome preparations were performed using an EpiScope nucleosome preparation kit (\#5333; Takara). Briefly, nuclei were isolated from control and Ubtf1/2 depleted cells and incubated at $37^{\circ} \mathrm{C}$ for $30 \mathrm{~min}$ with MNase. A control without MNase was included for each sample. gDNA was then isolated and subjected to qPCR using FAST SYBR Green dye on the StepOnePlus real-time PCR system (Applied Biosystems).
The relative level of MNase resistance was calculated after normalization to mock-digested DNA.

\section{Data access}

Raw ChIP-seq data is available through the NCBI Sequence Read Archive (SRA, http://www.ncbi.nlm.nih.gov/sra/) under accession number SRP039369. Processed ChIP-seq data and microarray expression data have been submitted to the NCBI Gene Expression Omnibus (GEO, http://www.ncbi.nlm.nih.gov/geo/) under accession numbers GSE63255 and GSE55461, respectively.

\section{Acknowledgments}

We thank Marcia Amalia for her technical contribution. This work was supported by grants from the National Health and Medical Research Council of Australia (NHMRC), the Leukaemia Foundation of Australia, Cancer Council Victoria, and the Captain Courageous Foundation. R.D.H. is supported by a NHMRC fellowship.T.M. is supported by funding from the Canadian Institutes of Health Research (CIHR) (\#MOP12205, http://www. cihr-irsc.gc.ca/e/193.html).

\section{References}

Anamika K, Gyenis A, Poidevin L, Poch O, Tora L. 2012. RNA polymerase II pausing downstream of core histone genes is different from genes producing polyadenylated transcripts. PLOS ONE 7: e38769.

Bartkova J, Horejsi Z, Koed K, Kramer A, Tort F, Zieger K, Guldberg P, Sehested M, Nesland JM, Lukas C, et al. 2005. DNA damage response as a candidate anti-cancer barrier in early human tumorigenesis. Nature 434: 864-870.

Berger AB, Decourty L, Badis G, Nehrbass U, Jacquier A, Gadal O. 2007. Hmo1 is required for TOR-dependent regulation of ribosomal protein gene transcription. Mol Cell Biol 27: 8015-8026.

Blahnik KR, Dou L, O'Geen H, McPhillips T, Xu X, Cao AR, Iyengar S, Nicolet CM, Ludascher B, Korf I, et al. 2010. Sole-Search: an integrated analysis program for peak detection and functional annotation using ChIP-seq data. Nucleic Acids Res 38: e13.

Boulon S, Westman B, Hutten S, Boisvert F, Lamond A. 2010. The nucleolus under stress. Mol Cell 40: $216-227$.

Bywater MJ, Poortinga G, Sanij E, Hein N, Peck A, Cullinane C, Wall M, Cluse L, Drygin D, Anderes K, et al. 2012. Inhibition of RNA polymerase I as a therapeutic strategy to promote cancer-specific activation of p53. Cancer Cell 22: 51-65.

Bywater MJ, Pearson RB, McArthur GA, Hannan RD. 2013. Dysregulation of the basal RNA polymerase transcription apparatus in cancer. Nat Rev Cancer 13: 299-314.

Castillo-Davis CI, Mekhedov SL, Hartl DL, Koonin EV, Kondrashov FA. 2002. Selection for short introns in highly expressed genes. Nat Genet 31: 415-418.

Cavanaugh A, Hirschler-Laszkiewicz I, Rothblum L. 2003. Ribosomal DNA transcription in mammals. In The Nucleolus (ed. Olson MOJ), pp. 89129. Kluwer Academic/Plenum, New York.

Chan JC, Hannan KM, Riddell K, Ng PY, Peck A, Lee RS, Hung S, Astle MV, Bywater M, Wall M, et al. 2011. AKT promotes rRNA synthesis and cooperates with c-MYC to stimulate ribosome biogenesis in cancer. $S c i$ Signal 4: ra56.

Chauvin C, Koka V, Nouschi A, Mieulet V, Hoareau-Aveilla C, Dreazen A Cagnard N, Carpentier W, Kiss T, Meyuhas O, et al. 2014. Ribosomal protein $\mathrm{S} 6$ kinase activity controls the ribosome biogenesis transcriptional program. Oncogene 33: 474-483.

Clemente-Ruiz M, Prado F. 2009. Chromatin assembly controls replication fork stability. EMBO Rep 10: 790-796.

Deisenroth C, Zhang Y. 2010. Ribosome biogenesis surveillance: probing the ribosomal protein-Mdm2-p53 pathway. Oncogene 29: 4253-4260.

Diesch J, Hannan RD, Sanij E. 2014. Perturbations at the ribosomal genes loci are at the centre of cellular dysfunction and human disease. Cell Biosci 4: 43.

Drygin D, Lin A, Bliesath J, Ho CB, O'Brien SE, Proffitt C, Omori M, Haddach M, Schwaebe MK, Siddiqui-Jain A, et al. 2011. Targeting RNA polymerase I with an oral small molecule CX-5461 inhibits ribosomal RNA synthesis and solid tumor growth. Cancer Res 71: 1418-1430.

Drygin D, O’Brien SE, Hannan RD, McArthur GA, Von Hoff DD. 2014 Targeting the nucleolus for cancer-specific activation of p53. Drug Discov Today 19: 259-265. 
Elenbaas B, Spirio L, Koerner F, Fleming MD, Zimonjic DB, Donaher JL, Popescu NC, Hahn WC, Weinberg RA. 2001. Human breast cancer cells generated by oncogenic transformation of primary mammary epithelial cells. Genes Dev 15: 50-65.

Fenech M. 2006. Cytokinesis-block micronucleus assay evolves into a "cytome" assay of chromosomal instability, mitotic dysfunction and cell death. Mutat Res 600: $58-66$.

Fuda NJ, Ardehali MB, Lis JT. 2009. Defining mechanisms that regulate RNA polymerase II transcription in vivo. Nature 461: 186-192.

Gentleman RC, Carey VJ, Bates DM, Bolstad B, Dettling M, Dudoit S, Ellis B, Gautier L, Ge Y, Gentry J, et al. 2004. Bioconductor: open software development for computational biology and bioinformatics. Genome Biol 5: R80.

Grob A, Colleran C, McStay B. 2014. Construction of synthetic nucleoli in human cells reveals how a major functional nuclear domain is formed and propagated through cell division. Genes Dev 28: 220-230.

Grueneberg DA, Pablo L, Hu KQ, August P, Weng Z, Papkoff J. 2003. A functional screen in human cells identifies UBF2 as an RNA polymerase II transcription factor that enhances the $\beta$-catenin signaling pathway. Mol Cell Biol 23: 3936-3950.

Grummt I. 1999. Regulation of mammalian ribosomal gene transcription by RNA polymerase I. Prog Nucleic Acid Res Mol Biol 62: 109-154.

Gunjan A, Paik J, Verreault A. 2005. Regulation of histone synthesis and nucleosome assembly. Biochimie 87: 625-635.

Hamdane N, Stefanovsky VY, Tremblay MG, Nemeth A, Paquet E, Lessard F, Sanij E, Hannan R, Moss T. 2014. Conditional inactivation of Upstream Binding Factor reveals its epigenetic functions and the existence of a somatic nucleolar precursor body. PLoS Genet 10: e1004505.

Han M, Chang M, Kim UJ, Grunstein M. 1987. Histone H2B repression causes cell-cycle-specific arrest in yeast: effects on chromosomal segregation, replication, and transcription. Cell 48: 589-597.

Hannan RD, Rothblum LI. 1995. Regulation of ribosomal DNA transcription during neonatal cardiomyocyte hypertrophy. Cardiovasc Res 30: 501-510.

Hannan R, Stefanovsky V, Arino T, Rothblum L, Moss T. 1999. Cellular regulation of ribosomal DNA transcription: both rat and Xenopus UBF1 stimulate rDNA transcription in 3T3 fibroblasts. Nucleic Acids Res 27: 1205-1213.

Hannan KM, Sanij E, Hein N, Hannan RD, Pearson RB. 2011. Signaling to the ribosome in cancer-it is more than just mTORC1. IUBMB Life 63: 79-85.

Hannan KM, Sanij E, Rothblum LI, Hannan RD, Pearson RB. 2013. Dysregulation of RNA polymerase I transcription during disease. Biochim Biophys Acta 1829: 342-360.

Hein N, Sanij E, Quin J, Hannan KM, Ganley ARD, Hannan RD. 2012. The nucleolus and ribosomal genes in aging and senescence. In Senescence (ed. Nagata T). InTech, Rijeka, Croatia. http://www.intechopen.com/ books/senescence/the-nucleolus-in-aging-and-senescence-

Hein N, Hannan KM, George AJ, Sanij E, Hannan RD. 2013. The nucleolus: an emerging target for cancer therapy. Trends Mol Med 19: 643-654.

Hovhannisyan H, Cho B, Mitra P, Montecino M, Stein GS, Van Wijnen AJ, Stein JL. 2003. Maintenance of open chromatin and selective genomic occupancy at the cell cycle-regulated histone $\mathrm{H} 4$ promoter during differentiation of HL-60 promyelocytic leukemia cells. Mol Cell Biol 23: $1460-1469$.

Irizarry RA, Bolstad BM, Collin F, Cope LM, Hobbs B, Speed TP. 2003. Summaries of Affymetrix GeneChip probe level data. Nucleic Acids Res 31: e15.

Laferte A, Favry E, Sentenac A, Riva M, Carles C, Chedin S. 2006. The transcriptional activity of RNA polymerase I is a key determinant for the level of all ribosome components. Genes Dev 20: 2030-2040.

Larson DE, Xie W, Glibetic M, O'Mahony D, Sells BH, Rothblum LI. 1993. Coordinated decreases in rRNA gene transcription factors and rRNA synthesis during muscle cell differentiation. Proc Natl Acad Sci 90: 79337936.

Li H, Durbin R. 2009. Fast and accurate short read alignment with BurrowsWheeler transform. Bioinformatics 25: 1754-1760.

Mais C, Wright JE, Prieto JL, Raggett SL, McStay B. 2005. UBF-binding site arrays form pseudo-NORs and sequester the RNA polymerase I transcription machinery. Genes Dev 19: 50-64.

Marzluff WF, Gongidi P, Woods KR, Jin J, Maltais LJ. 2002. The human and mouse replication-dependent histone genes. Genomics 80: 487-498.

Marzluff WF, Wagner EJ, Duronio RJ. 2008. Metabolism and regulation of canonical histone mRNAs: life without a poly(A) tail. Nat Rev Genet 9: 843-854.

McLean CY, Bristor D, Hiller M, Clarke SL, Schaar BT, Lowe CB, Wenger AM, Bejerano G. 2010. GREAT improves functional interpretation of cisregulatory regions. Nat Biotechnol 28: 495-501.

McStay B, Grummt I. 2008. The epigenetics of rRNA genes: from molecular to chromosome biology. Annu Rev Cell Dev Biol 24: 131-157.

Medina R, Ghule PN, Cruzat F, Barutcu AR, Montecino M, Stein JL, van Wijnen AJ, Stein GS. 2012. Epigenetic control of cell cycle-dependent histone gene expression is a principal component of the abbreviated pluripotent cell cycle. Mol Cell Biol 32: 3860-3871.

Meeks-Wagner D, Hartwell LH. 1986. Normal stoichiometry of histone dimer sets is necessary for high fidelity of mitotic chromosome transmission. Cell 44: 43-52.

Mendoza-Parra MA, Pattabhiraman S, Gronemeyer H. 2012. Sequential chromatin immunoprecipitation protocol for global analysis through massive parallel sequencing (reChIP-seq). Protoc Exch doi: 10.1038/ protex.2011.257.

Moss T, Stefanovsky VY. 2002. At the center of eukaryotic life. Cell 109: 545548.

Moss T, Langlois F, Gagnon-Kugler T, Stefanovsky V. 2007. A housekeeper with power of attorney: the rRNA genes in ribosome biogenesis. Cell Mol Life Sci 64: 29-49.

Myung K, Pennaneach V, Kats ES, Kolodner RD. 2003. Saccharomyces cerevisiae chromatin-assembly factors that act during DNA replication function in the maintenance of genome stability. Proc Natl Acad Sci 100: 6640-6645.

Naughton C, Avlonitis N, Corless S, Prendergast JG, Mati IK, Eijk PP, Cockroft SL, Bradley M, Ylstra B, Gilbert N. 2013. Transcription forms and remodels supercoiling domains unfolding large-scale chromatin structures. Nat Struct Mol Biol 20: 387-395.

O'Sullivan AC, Sullivan GJ, McStay B. 2002. UBF binding in vivo is not restricted to regulatory sequences within the vertebrate ribosomal DNA repeat. Mol Cell Biol 22: 657-668.

Phatnani HP, Greenleaf AL. 2006. Phosphorylation and functions of the RNA polymerase II CTD. Genes Dev 20: 2922-2936.

Pinto I, Winston F. 2000. Histone H2A is required for normal centromere function in Saccharomyces cerevisiae. EMBO J 19: 1598-1612.

Poortinga G, Hannan KM, Snelling H, Walkley CR, Jenkins A, Sharkey K, Wall M, Brandenburger Y, Palatsides M, Pearson RB, et al. 2004. MAD1 and c-MYC regulate UBF and rDNA transcription during granulocyte differentiation. EMBO J 23: 3325-3335.

Poortinga G, Wall M, Sanij E, Siwicki K, Ellul J, Brown D, Holloway TP, Hannan RD, McArthur GA. 2011. c-MYC coordinately regulates ribosomal gene chromatin remodeling and Pol I availability during granulocyte differentiation. Nucleic Acids Res 39: 3267-3281.

Poortinga G, Quinn LM, Hannan RD. 2014. Targeting RNA polymerase I to treat MYC-driven cancer. Oncogene doi: 10.1038/onc.2014.13.

Prado F, Aguilera A. 2005. Partial depletion of histone H4 increases homologous recombination-mediated genetic instability. Mol Cell Biol 25: 1526-1536.

Rao S, Procko E, Shannon MF. 2001. Chromatin remodeling, measured by a novel real-time polymerase chain reaction assay, across the proximal promoter region of the IL-2 gene. J Immunol 167: 4494-4503.

Sanij E, Hannan RD. 2009. The role of UBF in regulating the structure and dynamics of transcriptionally active rDNA chromatin. Epigenetics 4: 374-382.

Sanij E, Poortinga G, Sharkey K, Hung S, Holloway TP, Quin J, Robb E, Wong LH, Thomas WG, Stefanovsky V, et al. 2008. UBF levels determine the number of active ribosomal RNA genes in mammals. J Cell Biol 183: 1259-1274.

Smyth G. 2005. Limma: linear models for microarray data. In Bioinformatics and computational biology solutions using $R$ and Bioconductor (ed. Gentleman R, et al.), pp. 397-420. Springer, New York.

Stefanovsky VY, Moss T. 2008. The splice variants of UBF differentially regulate RNA polymerase I transcription elongation in response to ERK phosphorylation. Nucleic Acids Res 36: 5093-5101.

Stefanovsky VY, Pelletier G, Bazett-Jones DP, Crane-Robinson C, Moss T. 2001. DNA looping in the RNA polymerase I enhancesome is the result of non-cooperative in-phase bending by two UBF molecules. Nucleic Acids Res 29: 3241-3247.

Stefanovsky V, Langlois F, Gagnon-Kugler T, Rothblum LI, Moss T. 2006. Growth factor signaling regulates elongation of RNA polymerase I transcription in mammals via UBF phosphorylation and r-chromatin remodeling. Mol Cell 21: 629-639.

White RJ. 2008. RNA polymerases I and III, non-coding RNAs and cancer. Trends Genet 24: 622-629.

Ye X, Franco AA, Santos H, Nelson DM, Kaufman PD, Adams PD. 2003. Defective $S$ phase chromatin assembly causes DNA damage, activation of the S phase checkpoint, and S phase arrest. Mol Cell 11: 341-351.

Zentner GE, Saiakhova A, Manaenkov P, Adams MD, Scacheri PC. 2011. Integrative genomic analysis of human ribosomal DNA. Nucleic Acids Res 39: 4949-4960.

Zhang Y, Liu T, Meyer CA, Eeckhoute J, Johnson DS, Bernstein BE, Nussbaum C, Myers RM, Brown M, Li W, et al. 2008. Model-based analysis of ChIP-Seq (MACS). Genome Biol 9: R137.

Zhu LJ, Pages H, Gazin C, Lawson N, Lin S, Lapointe D, Green M. 2009. ChIPpeakAnno: Batch annotation of the peaks identified from either ChIP-seq or ChIP-chip experiments. R package version 1.6.0.

Received March 26, 2014; accepted in revised form November 26, 2014.

\section{Genome Research}




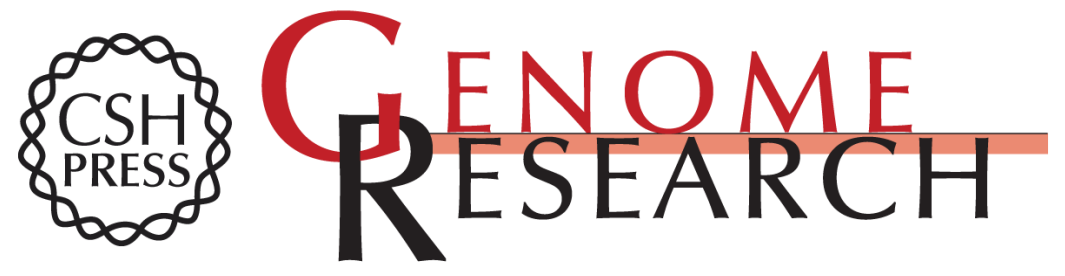

\section{A novel role for the Pol I transcription factor UBTF in maintaining genome stability through the regulation of highly transcribed Pol II genes}

Elaine Sanij, Jeannine Diesch, Analia Lesmana, et al.

Genome Res. 2015 25: 201-212 originally published online December 1, 2014

Access the most recent version at doi:10.1101/gr.176115.114

\section{Supplemental http://genome.cshlp.org/content/suppl/2014/12/15/gr.176115.114.DC1 \\ Material}

References This article cites 64 articles, 20 of which can be accessed free at:

http://genome.cshlp.org/content/25/2/201.full.html\#ref-list-1

Open Access Freely available online through the Genome Research Open Access option.

Creative This article, published in Genome Research, is available under a Creative

Commons Commons License (Attribution-NonCommercial 4.0 International), as described at

License http://creativecommons.org/licenses/by-nc/4.0/.

Email Alerting Receive free email alerts when new articles cite this article - sign up in the box at the Service top right corner of the article or click here.

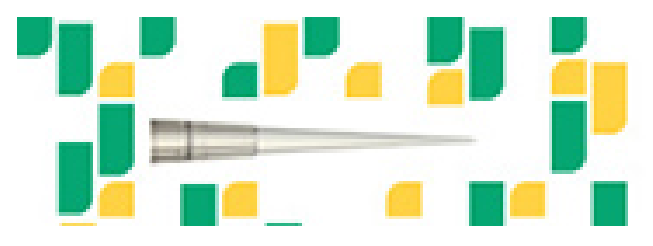

Focused on your science.

To subscribe to Genome Research go to:

https://genome.cshlp.org/subscriptions 